\title{
Guide de pratique clinique 2020 de l'ACO : Réadaptation optométrique de la basse vision Sommaire
}

\author{
Les membres du Groupe \\ de travail du GPC sur la basse \\ vision sont :
}

Susan J. Leat, B.Sc. (spécialisé), PhD, FCOptom, FAAO

École d'optométrie et des sciences de la vision, Université de Waterloo

\section{Alexis Keeling, B.Sc., B.Sc.Ed, OD \\ $\mathrm{D}^{\mathrm{r}}$ Alexis Keeling Optometry}

Tammy Labreche, B.Sc. (spécialisé), OD, FAAO École d'optométrie et des sciences de la vision, Université de Waterloo

Julie-Andrée Marinier, B.Sc., OD, M.Sc.

École d'optométrie, Université de Montréal

Rajan Mistry, B.Sc. (spécialisé), OD Alberta Eye Health Clinic

Michael Nelson, OD, FAAO Waverley Eye Care Centre
Abraham Yuen, B.Sc. (spécialisé), OD FYidoctors Markham/ Richmond University Vision Care

\begin{abstract}
OBJET
Le Guide de pratique clinique en basse vision a pour but d'aider les optométristes canadiens à fournir des soins optimaux de réadaptation aux patients atteints de déficience visuelle. Le Guide de pratique est fondé sur les meilleures données probantes disponibles, interprétées par un groupe d'experts. Le groupe de rédaction est composé d'optométristes du milieu universitaire et de la pratique privée, représentant diverses régions du Canada. Le Guide de pratique aidera les optométristes à identifier les patients qui ont besoin d'une réadaptation de la basse vision. Il recommande une évaluation et une prise en charge appropriées.
\end{abstract}

\section{INTRODUCTION}

\section{Le problème}

À l'heure actuelle, on estime que $0,95 \%$ de la population canadienne souffre d'une déficience visuelle (basse vision, cécité, etc ${ }^{1}$.) La prévalence augmente de façon exponentielle avec l'âge ${ }^{2}$. Entre 75 et 84 ans, le pourcentage de personnes souffrant d'une déficience visuelle atteint environ 6 à 8,9\% \% . La population canadienne vieillit ${ }^{4}$; d'ici 2036, les personnes âgées devraient représenter de 23 à $25 \%$ de la population. Il y aura donc une augmentation proportionnelle du nombre de personnes atteintes de déficience visuelle ${ }^{1,3,5,6}$. Le nombre de personnes ayant une basse vision devrait augmenter de plus du double au cours des 30 prochaines années ${ }^{2}$. Le besoin de réadaptation de la basse vision (RBV) est de plus en plus urgent pour réduire les effets de la déficience visuelle.

La déficience visuelle entraîne des répercussions considérables et de grande envergure. Elle est associée à une incapacité (limitations d'activité) pour la mobilité et pour les tâches visuellement intensives (lecture, écriture) ${ }^{7}$. Elle est également cause de défaillance dans l'exécution des tâches de la vie quotidienne, comme les soins personnels, les courses et la préparation des repas, par rapport à la population générale et aux personnes atteintes d'autres maladies chroniques ${ }^{7-9}$. Les personnes souffrant d'une déficience visuelle sont moins susceptibles d'avoir un emploi et plus isolées sur le plan social, elles ont aussi plus de problèmes de transport et risquent davantage de faire une chute. Elles sont plus sujettes à la dépression, à d'autres problèmes de santé mentale, à une réduction globale de leur qualité de vie et à un risque plus élevé de mortalité $8,10-12$.

\section{Modèle de réadaptation de la basse vision}

Des études ont prouvé l'efficacité de la $\mathrm{RBV}^{13,14}$ fournie par les optométristes en milieu communautaire ou en privé ${ }^{15-17}$, y compris pour les patients présentant une perte de vision mineure ${ }^{15}$. Les optométristes canadiens sont idéalement qualifiés ${ }^{18}$ pour jouer un rôle déterminant dans la prestation de traitements de la basse vision, et ils exercent dans des contextes multidisciplinaires ou interdisciplinaires intégraux où ils effectuent l'évaluation initiale, réalisent les premières interventions et mettent au point un plan de réadaptation. Vu l'immense superficie du Canada et la dispersion de sa population rurale, il est essentiel pour assurer un accès équitable aux services que les optométristes jouent un rôle clé dans la $\mathrm{RBV}^{19}$. 


\section{Niveau 1. RBV}

Ce Guide de pratique clinique adopte un modèle à trois niveaux de RBV. Il énonce qu'à titre de norme minimale de soins, il incombe à tous les optométristes de fournir directement des services de RBV ou de recommander de tels services par un optométriste spécialiste de la basse vision, avant tout aiguillage vers d'autres organismes. Ceci constitue une RBV de niveau 1 qui devrait avoir lieu dès que le patient souffre d'une déficience visuelle permanente. Lorsqu'il est avéré qu'un degré de perte de vision est irréversible, la RBV devrait constituer un processus parallèle au traitement de la maladie oculaire.

L'évaluation et la réadaptation en basse vision devraient toujours être recommandées dans les cas suivants :

- Un patient ayant une basse vision qui est définie comme étant une déficience visuelle (perte mesurable de la vision) entraînant une incapacité visuelle (difficulté à entreprendre une tâche en raison d'une mauvaise vision).

- À titre de précision, sont comprises toutes les pathologies suivantes :

o une maladie ou blessure incurable (oculaire ou systémique) pour laquelle un traitement chirurgical ou médical a été entrepris, envisagé ou est en cours;

ET

o une réduction de la vision corrigée (le plus souvent atteinte de l'acuité visuelle, de la sensibilité différentielle ou des champs visuels) par rapport aux normes de l'âge;

ET

- une difficulté à accomplir les tâches visuelles souhaitées en dépit d'une correction visuelle optimale.

- En ce qui a trait à la déficience visuelle, les niveaux auxquels la perte de vision est susceptible de causer une incapacité visuelle sont les suivants, sans toutefois s'y limiter :

o acuité visuelle (AC) de 6/12 (20/40) ou inférieure;

OU

- scotome central ou paracentral ou métamorphopsie;

OU

o perte périphérique de champ (hémianopsie ou hémianopsie en quadrant; champ visuel circulaire total de moins de 70 degrés $\left.^{1}\right)$;

OU

- $\log \mathrm{CS}<1,4$

Évaluation supplémentaire minimale : Afin d'assurer un aiguillage approprié, il est important de vérifier les incapacités autodéclarées d'un patient, sa vision fonctionnelle et ses objectifs. Il est important que la réfraction soit exacte (idéalement avec des montures d'essai) et que la mesure de l'acuité visuelle corrigée soit la meilleure possible. Tous les optométristes devraient être disposés et aptes à tester des additions plus élevées pour la lecture (jusqu'à 4D). Il est fortement recommandé de procéder à une évaluation des champs visuels et de la sensibilité différentielle pour recueillir les renseignements nécessaires à un aiguillage approprié.

Au-delà de cette exigence, il y a deux niveaux auxquels les optométristes peuvent choisir de fournir des services de RBV.

\section{Niveau 2. RBV de base}

Ce niveau de RBV peut être fourni dans le bureau d'un optométriste avec une gamme réduite d'équipements et de dispositifs optiques et, idéalement, avec le soutien d'un technicien ou aide-optométriste qualifié, ou d'un thérapeute en basse vision.

Les patients qui sont susceptibles d'en bénéficier sont ceux qui présentent les symptômes suivants :

- $\quad \mathrm{AV}$ de 6/12 à 6/21 inclusivement et/ou sensibilité différentielle Log entre 1,40 et 1,00;

- aucune hémianopsie ou hémianopsie en quadrant, et champ visuel circulaire de plus de 70 degrés;

- aucune perte importante de champ périphérique qui limite la vitesse de lecture ou la fonction visuelle.

\section{Niveau 3. RBV global}

Les patients dont la vision est plus faible que celle indiquée ci-dessus pour le RBV de base de niveau 2 auront probablement besoin de toute la gamme d'appareils optiques et électroniques, ainsi que des services décrits dans le Guide de pratique clinique de basse vision ${ }^{20}$, résumé ci-dessous. 


\section{RÉADAPTATION OPTOMÉTRIQUE DE LA BASSE VISION}

La réadaptation de la basse vision commence par une évaluation complète et approfondie de la fonction visuelle et un examen des maladies oculaires et des problèmes de santé systémiques qui peuvent avoir un effet sur cette fonction (capacité mesurée du système visuel) et sur la vision fonctionnelle (capacité d'entreprendre les tâches de la vie quotidienne liées à la vision ${ }^{21}$. Il en résulte la création d'un plan initial de réadaptation de la basse vision. La gestion de la réadaptation de la basse vision comprend l'évaluation du patient et la formation au moyen de diverses aides optiques ou non optiques à la basse vision, de diverses stratégies de réadaptation axées sur les besoins particuliers, et d'éducation et de conseils à l'intention des patients. Le résultat est le plan de réadaptation qui contient les recommandations finales pour le patient.

\section{Évaluation de la basse vision}

Un historique complet du cas est effectué avec attention particulière sur les incapacités et les objectifs autodéclarés du patient, notamment un examen des domaines fonctionnels, activités de la vie quotidienne, activités sociales, et exigences professionnelles, éducatives ou de loisirs qui peuvent être touchés par la déficience visuelle. Il faut ensuite établir l'ordre de priorité des objectifs avec le patient. L'étude de cas doit également porter sur l'efficacité des lunettes et des appareils actuels, les antécédents oculaires, familiaux, sociaux, de santé en général, de consommation de médicaments et de chutes, ainsi que sur les effets de l'éblouissement et de l'éclairage, la stabilité de l'état oculaire et la compréhension du patient lui-même de son état de santé oculaire et des conséquences qui en découlent.

La réfraction avec les montures d'essai (objective suivie de subjective) à l'aide de changements de lentille fondés sur le seuil différentiel est un élément essentiel de l'évaluation de la basse vision. Il est souvent possible d'obtenir une amélioration importante de l'AV ${ }^{22,23}$ et le discernement de la réfraction exacte est un facteur essentiel pour l'exactitude de l'évaluation et de la prescription de la plupart des appareils optiques. Les acuités visuelles à distance habituelles et corrigées, de préférence à l'aide de tableaux d'acuité visuelle basés sur les principes du logMAR (l'angle minimum de résolution) et conçus pour la basse vision ${ }^{24}$ et adaptés au niveau de vision et à l'âge du patient ${ }^{24}$. Il faudrait idéalement mesurer l'acuité visuelle de près à l'aide des tableaux à texte continu du logMAR, et enregistrer la vision à distance et la lecture de mots imprimés. Il faudrait également tenir compte des effets de l'éclairage sur l'acuité visuelle.

La sensibilité différentielle est une mesure importante pour évaluer la fonction visuelle ou une incapacité du patient et pour prédire le résultat avec un grossissement ${ }^{25,26}$. Elle permet de prédire les difficultés d'une vaste gamme d'autres tâches visuelles (aptitudes à la vie quotidienne, mobilité, discrimination du visage, conduite) et incapacité perçue $^{7,27-30}$. Une faible sensibilité différentielle est également un facteur de risque de chute ${ }^{31,32}$. Il faut tenir compte de la perte du champ visuel (central ou périphérique) et la mesurer régulièrement. Pour déterminer la perte de fonction due à la maladie oculaire du patient, il peut être indiqué de procéder à des examens supplémentaires, tels que la vision des couleurs et les tests d'éblouissement. L'évaluation de la santé oculaire permet au clinicien de constater tout progrès de la maladie et la contribution de multiples causes à une basse vision. L'examen du fond de l'œil dilaté n'est pas systématiquement inclus dans une évaluation de la basse vision, car il est habituellement effectué auparavant. Par contre, il peut être nécessaire dans les cas où les symptômes, les incapacités ou d'autres mesures ne correspondent pas au diagnostic actuel ou en l'absence d'examen récent de la santé oculaire. Il faudra habituellement un rendez-vous distinct pour l'examen du fond de l'œil dilaté.

À la fin de l'évaluation, l'optométriste est en mesure de créer un plan de réadaptation de la basse vision. L’optométriste spécialiste de la basse vision et son assistant peuvent mettre en place de nombreuses composantes. La mise en œuvre du plan complet peut nécessiter l'aiguillage vers d'autres fournisseurs de services. Après une détermination de la prise en charge appropriée du patient (y compris les aiguillages), décrite ci-dessous, le plan de réadaptation fait l'objet d'une révision.

\section{Gestion de la basse vision}

Les outils à la disposition du clinicien en basse vision comprennent le grossissement optique, non optique et électronique, l'augmentation du contraste, le contrôle de l'éclairage, la minimisation, le déplacement de l'objet ou de l'image, la formation et les adaptations. Constituent d'autres éléments importants l'éducation des patients et de leur famille et l'aiguillage vers d'autres fournisseurs de services.

Les patients atteints d'une perte de vision centrale peuvent être pris en charge par grossissement optique et électronique, selon les besoins pour les tâches éloignées, semi-éloignées et rapprochées. Le grossissement optique pour les besoins relatifs aux tâches proches ou semi-éloignées se fait au moyen de prismes élevés, de microscopes, de télémicroscopes et de loupes à main et sur pied, et pour les tâches à distance, au moyen de télescopes. De nombreux appareils d'assistance optique peuvent être personnalisés pour tenir compte de l'erreur de réfraction d'un patient. Il faudrait également envisager pour de nombreux patients le grossissement électronique et les options d'accessibilité de la technologie traditionnelle. Le grossissement électronique se fait au moyen de loupes à main, portables et de 
bureau. Il est efficace pour les patients qui présentent une perte de sensibilité différentielle ou d'importants scotomes centraux. Les patients peuvent souvent bénéficier à la fois du grossissement optique et électronique. Les patients ont tendance à utiliser plus fréquemment les loupes optiques pour une variété de tâches, et à préférer le grossissement électronique pour lire plus longtemps, lire des caractères plus petits et pour leur lecture de loisir ${ }^{33,34}$. Les fonctions d'accessibilité des appareils actuels (téléphone mobile, tablette, ordinateur portatif ou de bureau) comprennent des options de texte, d'assistance vocale, de grossissement, de contraste, de police et de couleur.

Bien qu'il y ait peu de preuves de l'efficacité de l'entraînement à la vision excentrique ${ }^{35}$, elle continue de faire partie de l'éventail des solutions pour les patients atteints de scotomes centraux.

La sensibilité différentielle réduite est l'autre principale catégorie de troubles de la vision causant une incapacité parallèle à la perte de la vision centrale (perte d'AV) et des troubles du champ visuel. Lorsque la sensibilité différentielle est réduite à moins de $1,40^{7,29}$, le patient risque d'avoir certaines incapacités, comme des problèmes de mobilité et de résolution, mais lorsqu'elle est inférieure à 1,00, la performance visuelle est gravement compromise, même avec un grossissement approprié. Par exemple, la lecture est probablement lente, même avec l'aide de loupes optiques ${ }^{25,26,36}$.

Les approches pour la perte de sensibilité différentielle comprennent a) la modification de la sensibilité différentielle du patient par une manipulation de l'éclairage, l'essai de filtres ou d'un typoscope, ou l'inversion du contraste sur un grossissement électronique ou b) l'augmentation du contraste de la tâche avec des dispositifs électroniques, des modifications de l'environnement et l'utilisation de méthodes de substitution de la vue, par exemple, la sortie vocale sur un ordinateur.

La gestion de la perte de vision périphérique comprend l'utilisation de prismes pour l'hémianopsie (des prismes sectoriels ou des prismes de Peli), de prismes sectoriels ou de minimiseurs pour les champs resserrés, la formation à la recherche visuelle, les stratégies pour améliorer l'orientation visuelle pendant la lecture, l'aiguillage vers une orientation et une formation sur la mobilité.

Les patients atteints de nystagmus peuvent bénéficier de yoked prisms et du positionnement des tâches pour permettre l'utilisation confortable de leur point nul. Il est possible d'améliorer l'AV de certains patients atteints de nystagmus infantile au moyen de lentilles cornéennes ${ }^{37,38}$.

Les niveaux d'éclairage peuvent améliorer considérablement la fonction des patients ayant une déficience visuelle et il convient d'en faire l'essai. Il peut être très avantageux pour de nombreux patients d'utiliser les filtres de transmission sélectifs et non sélectifs pour contrôler les niveaux de lumière et d'éblouissement et d'optimiser leur confort. Les teintes jaunes de courte longueur d'onde offrent souvent des avantages subjectifs pour les patients, bien qu'il n'y ait actuellement aucune preuve objective qu'elles améliorent l'AV, la lecture ou la sensibilité différentielle chez les personnes ayant une déficience visuelle ${ }^{34}$. Les lentilles cornéennes teintées ou irisées peuvent être bénéfiques pour les patients souffrant de photophobie extrême.

Pour tous les appareils et toutes les recommandations de réadaptation, la sélection devrait suivre un processus décisionnel axé sur le patient, c.-à-d. le meilleur dispositif pour la ou les tâches du patient, en fonction de sa propre appréciation.

L'optométriste devrait être en mesure de recommander des appareils non optiques appropriés, comme des livres, horloges et montres à gros caractères, des appareils avec sortie auditive, p. ex., livres et moniteurs de glycémie sonores, et des approches tactiles comme le marquage des appareils. Il est important pour le progrès des patients de communiquer et de collaborer avec les autres professionnels de l'équipe de réadaptation. Lorsque la situation le justifie, l'optométriste devrat aiguiller le patient vers les autres services comme la formation à l'orientation et la mobilité, l'ergothérapie, la thérapie contre la basse vision, les évaluations de haute technologie, les services sociaux et communautaires, le counseling, les services de consultation en génétique, les conseils professionnels et la consultation chirurgicale au besoin, p. ex., cataracte, nystagmus, strabisme. Au moment de l'aiguillage, il est recommandé que l'optométriste inclue son plan de réadaptation, ainsi que les interventions qui ont été envisagées et mises en œuvre.

\section{CONCLUSION}

La RBV nécessite une approche holistique à l'égard du patient, et l'optométriste doit être conscient de son état émotionnel et psychologique. Les interventions qui sont recommandées doivent être particulières non seulement aux tâches, mais aussi au patient lui-même, c.-à-d. adaptées à ses objectifs, ses exigences et ses limites. La RBV est un processus continu pour la plupart des patients et il est important d'assurer un suivi, car le niveau d'acceptation, les activités et les objectifs des patients peuvent changer au fil du temps. 
Les optométristes sont particulièrement qualifiés pour fournir de la RBV, car ils réfractent de façon experte, optimisent la fonction visuelle avec des lunettes et des lentilles cornéennes, évaluent avec précision la fonction visuelle, comprennent les effets des problèmes oculaires, élaborent un plan de réadaptation visuelle, prescrivent des appareils optiques et non optiques, portatifs et montés sur des lunettes, fournissent une formation sur la vision et les appareils fonctionnels, donnent des conseils sur les stratégies visuelles et les modifications de l'environnement et coordonnent leurs interventions avec les autres services. •

\section{NOTE EN FIN DE TEXTE}

${ }^{1}$ Cela comprend 60 degrés, ce qui est le niveau pour obtenir un financement au Québec.

\section{RÉFÉRENCES}

1. Maberley D, Hollands H, Chuo J, et al. The prevalence of low vision and blindness in Canada. Eye. 2006;20:341-346.

2. Chan T, Friedman DS, Bradley C, Massof R. Estimates of incidence and prevalence of visual impairment, low vision, and blindness in the United States. JAMA Ophthalmol. 2018;136:12-19.

3. Aljied R, Aubin M-, Buhrmann R, Sabeti S, Freeman EE. Prevalence and determinants of visual impairment in Canada: cross-sectional data from the Canadian Longitudinal Study on Aging. Can J Ophthalmol. 2018;53:291-297.

4. Statistics Canada. An Aging Population. https://www150.statcan. gc.ca/nl/pub/11-402-x/2010000/chap/pop/pop02-eng.htm. Accessed March/14, 2019.

5. Attebo K, Mitchell P, Smith W. Visual acuity and the causes of visual loss in Australia: The Blue Mountains Eye Study. Ophthalmology. 1996;103:357-364.

6. Rubin GS, West SK, Muñoz B, et al. A comprehensive assessment of visual impairment in a population of older Americans: The SEE Study. Invest Ophthalmol Vis Sci. 1997;38:557-568.

7. West SK, Rubin GS, Broman AT, Munoz B, Bandeen-Roche K, Turano K. How does visual impairment affect performance on tasks of everyday life? The SEE Project. Salisbury Eye Evaluation. Arch Ophthalmol. 2002;120:774-780.

8. Kempen GIJM, Ballemans J, Ranchor AV, Van Rens GHMB, Zijlstra GAR. The impact of low vision on activities of daily living, symptoms of depression, feelings of anxiety and social support in community-living older adults seeking vision rehabilitation services. Qual Life Res. 2012;21:1405-1411.

9. Horowitz A. The prevalence and consequences of vision impairment in later life. Top Geriatr Rehabil. 2004;20:185-195.

10. Elliott DB. The Glenn A. Fry award lecture 2013: Blurred vision, spectacle correction, and falls in older adults. Optom Vis Sci. 2014;91:593-601.

11. Senra H, Barbosa F, Ferreira P, et al. Psychologic adjustment to irreversible vision loss in adults: A systematic review. Ophthalmology. 2015;122:851-861.

12. Zheng D, Christ SL, Lam BL, Arheart KL, Galor A, Lee DJ. Increased mortality risk among the visually impaired: The roles of mental well-being and preventive care practices. Invest Ophthalmol Vis Sci. 2012;53:2685-2692.

13. Binns AM, Bunce C, Dickinson C, et al. How effective is low vision service provision? A systematic review. Surv Ophthalmol. 2012;57:34-65.

14. Stelmack JA, Tang XC, Reda DJ, Rinne S, Mancil RM, Massof RW. Outcomes of the veterans affairs low vision intervention trial (LOVIT). Arch Ophthalmol. 2008;126:608-617.

15. Stelmack JA, Tang XC, Wei Y, et al. Outcomes of the veterans affairs low vision intervention trial II (LOVIT II) a randomized clinical trial. JAMA Ophthalmol. 2017;135:96-104

16. Court H, Ryan B, Bunce C, Margrain TH. How effective is the new community-based Welsh low vision service?. Br J Ophthalmol. 2011;95:178-184.

17. De Boer MR, Twisk J, Moll AC, Völker-Dieben HJ, De Vet HC, Van Rens GH. Outcomes of low-vision services using optometric and multidisciplinary approaches: a non-randomized comparison. Ophthal Physiol Opt. 2006;26:535-544.

18. Association of Schools and Colleges of Optometry. Entry-level competencies and learning objectives in visual impairment and low vision rehabilitation. https://optometriceducation.org/files/EntryLevelCompetencies_LowVision.pdf. Accessed April 16th, 2019.

19. Leat SJ. Proposed model for integrated low-vision rehabilitation services in Canada. Optom Vis Sci. 2016;93:77-84.

20. Leat SJ, Keeling A, Labreche T, et al. Canadian Association of Optometry. 2020 CAO Clinical Practice Guideline: Optometric Low Vision Rehabilitation. https://opto.ca/. Accessed Dec 17th 2019.

21. Colenbrander A. Visual functions and functional vision. Int Cong Ser. 2005;1282:482-486.

22. Leat SJ, Rumney NJ. The experience of a university-based low vision clinic. Ophthal Physiol Opt. 1990;10:8-15.

23. Sunness JS, El Annan J. Improvement of visual acuity by refraction in a low-vision population. Ophthalmol. 2010;117:1442-1446.

24. Bailey IL, Lovie-Kitchin JE. Visual acuity testing. From the laboratory to the clinic. Vis Res. 2013;90:2-9.

25. Whittaker SG, Lovie-Kitchin J. Visual requirements for reading. Optom Vis Sci. 1993;70:54-65.

26. Leat SJ, Woo GC. The validity of current clinical tests of contrast sensitivity and their ability to predict reading speed in low vision. Eye. 1997;11:893-899.

27. Rubin GS, Bandeen-Roche K, Huang GH, et al. The association of multiple visual impairments with self-reported visual disability: SEE project. Invest Ophthalmol Vis Sci. 2001;42:64-72.

28. Bowers A. Contrast sensitivity losses impair pedestrian detection more than visual acuity losses. https://www.aaopt.org/detail/knowledge-base-article/contrast-sensitivity-losses-impair-pedestriandetection-more-than-visual-acuity-losses. Accessed May 29th, 2019.

29. Rubin G, Rocher K, Prasda-Rao P, Fried L. Vision impairment and disability in older adults . Optom Vis Sci. 1994;71:750-760.

30. Barnes CS, De L'Aune W, Schuchard RA. A test of face discrimination ability in aging and vision loss. Optom Vis Sci. 2011;88:188-199.

31. Kuyk T, Elliott JL, Fuhr PS. Visual correlates of mobility in real world settings in older adults with low vision. Optom Vis Sci. 1998;75:538-547.

32. Lord SR. Visual risk factors for falls in older people. Age Ageing. 2006;35:42-45.

33. Taylor JJ, Bambrick R, Brand A, et al. Effectiveness of portable electronic and optical magnifiers for near vision activities in low vision: a randomised crossover trial. Ophthal Physiol Opt. 2017;37:370-384.

34. Virgili G, Acosta R, Bentley SA, Giacomelli G, Allcock C, Evans JR. Reading aids for adults with low vision. Cochrane Database Syst Rev. 2018; (4), CD003303.

35. Gaffney AJ, Margrain TH, Bunce CV, Binns AM. How effective is eccentric viewing training? A systematic literature review. Ophthal Physiol Opt. 2014;34:427-437.

36. Latham K, Tabrett D. Guidelines for Predicting Performance with Low Vision Aids. Optom Vis Sci. 2012;89:1316-1326.

37. Bagheri A, Abbasi H, Tavakoli M, Sheibanizadeh A, Kheiri B, Yazdani S. Effect of rigid gas permeable contact lenses on nystagmus and visual function in hyperopic patients with infantile nystagmus syndrome. Strabismus. 2017;25:17-22.

38. Jayaramachandran P, Proudlock FA, Odedra N, Gottlob I, McLean RJ. A randomized controlled trial comparing soft contact lens and rigid gas-permeable lens wearing in infantile nystagmus. Ophthalmol. 2014;121:1827-1836. 